\title{
Geoportal al servicio de la educación en Ecuador: GEOEDUCA*
}

\author{
Fernanda León-Pazmiño* \\ Rocío Narváez-Benalcázar $^{* *}$
}

Recibido el 14 de marzo de 2017; aceptado el 22 de mayo de 2017

\begin{abstract}
At present, the Spatial Data Infrastructures (SDI) have made available to all, through the Geoportals, a large amount of geographic information (GI), which until some years ago was very difficult to access.

On the other hand, the technological revolution with which the children of the last decades have grown, have prepared them to learn in a different way and thus to take advantage of the resources in the Network that are available; However, the adequacy of the information found cannot always be guaranteed.

With these premises in mind and aware of the importance of official geographic information, in the educational field, the Military Geographic Institute (IGM) of Ecuador launched a project called GEOEDUCA, which shows the experience of its development, implementation and use. This is dedicated to school students, as well as for teachers and parents providing access to the GI of the country. GEOEDUCA, named for its purpose of creation, is a sample of the various users that can have geoportals.
\end{abstract}

Key words: Geoportal, education, children, Ecuador.

\section{Resumo}

As Infraestruturas de Dados Espaciais (IDE), têm colocado ao alcance de todos, através dos Geoportais, grande quantidade de informação geográfica (IG) que até há alguns anos, era muito difícil de se acessar. Por outro lado, a revolução tecnológica com que tem crescido as crianças nas últimas décadas, os tem preparado para

* GEOEDUCA obtuvo el Premio GEOSUR Sexta edición, año 2017.

** Instituto Geográfico Militar (IGM), Seniergues E4-676 y Gral. Telmo Paz y Miño, El Dorado, Quito, Ecuador, correos electrónicos: fernanda.leon@igm.gob.ec, rocio.narvaez@igm.gob.ec 
aprender de maneira diferente aproveitando, entre outros meios, os recursos nas redes que se encontrem disponíveis; entretanto, nem sempre se pode garantir a idoneidade da informação encontrada.

Com estas premissas o Instituto Geográfico Militar do Equador (IGM), consciente da importância que tem a informação geográfica oficial, não só nos ambientes profissionais e políticos, se não também no âmbito educativo, lançou um projeto denominado GEOEDUCA, onde se mostra a experiência de seu desenvolvimento, implementação e uso. Este espaço web está dedicado a crianças e jovens em idade escolar, assim como para professores e pais que podem ter acesso a IG do país. GEOEDUCA, denominado assim por seu objetivo de criação, é uma mostra dos diversos usuários que podem ter os geoportais.

Palavras chave: Geoportal, educação, crianças, Equador.

\section{Resumen}

Las Infraestructuras de Datos Espaciales (IDE), han puesto al alcance de todos, a través de los Geoportales, gran cantidad de información geográfica (IG) a la que hasta hace algunos años, era muy difícil acceder. Por otro lado, la revolución tecnológica con la que han crecido los niños de las últimas décadas, los ha preparado para aprender de manera diferente aprovechando, entre otros medios, los recursos en la Red que se encuentren disponibles; sin embargo, no siempre se puede garantizar la idoneidad de la información encontrada.

Con estas premisas el Instituto Geográfico Militar del Ecuador (IGM), consciente de la importancia que tiene la información geográfica oficial, no solo en los ambientes profesionales y políticos, sino también en el ámbito educativo, puso en marcha un proyecto denominado GEOEDUCA, donde se muestra la experiencia de su desarrollo, implementación y uso. Este espacio web está dedicado a niños y jóvenes en edad escolar, así como para profesores y padres a quienes brinda acceso a la IG del país. GEOEDUCA, denominado así por su objetivo de creación, es una muestra de los diversos usuarios que pueden tener los geoportales.

Palabras clave: Geoportal, educación, niños, Ecuador.

\section{GEOEDUCA, el Geoportal orientado a la Educación}

La importancia de compartir la IG a nivel local, regional e incluso mundial, está determinada por la necesidad cada vez mayor de satisfacer la demanda de los distintos tipos de usuarios (González, 2010:5). Son los Geoportales de la IDE los que deben cumplir ese objetivo. El acceso a la IG ha ampliado en gran medida sus aplicaciones y por supuesto sus usuarios. Un grupo, que generalmente no es tomado en cuenta son los estudiantes escolares, los denominados "nativos digitales", quienes se ven bombardeados de datos que pueden ser de utilidad en su proceso de aprendizaje y para quienes la tecnología es parte de sus vidas. 
Recibir, intercambiar, elaborar y difundir información oficial, forma parte del derecho a la información de los ciudadanos y se convierte en un gran instrumento de construcción de futuro, en beneficio del fortalecimiento de la identidad, la inclusión y la convivencia. Bajo estas premisas se construyó el portal geográfico web GEOEDUCA, concebido como el espacio de aprendizaje, con el fin de poner a disposición de la comunidad educativa información geográfica-cartográfica oficial, que permitiera descubrir el país y su entorno.

En el Ecuador, el desarrollo de las Tecnologías de la Información y las Comunicaciones (TIC) y la facilidad de acceso a la IG sumado a factores como: a) la gran cantidad de información geográfica que produce no solo el IGM, que es el responsable de la información geográfica básica del país, sino distintas instituciones públicas (León et al., 2016) y b) la existencia de un marco legal que garantiza el acceso gratuito a la misma (LOTAIP, 2004), impulsaron la creación de este proyecto.

El objetivo principal de GEOEDUCA es garantizar el acceso a través de la red de Internet a la información geográfica oficial del país para ser usada con fines educativos e implementada para tres grupos de usuarios: estudiantes de educación básica, jóvenes de bachillerato y profesores.

Los contenidos considerados, abarcan las asignaturas de Entorno Natural y Social y Ciencias Sociales, donde se encuentran las temáticas geográficas principales con base en las mallas curriculares aprobadas por el Ministerio de Educación del país y disponibles a través de su sitio web.

El grupo de trabajo que diseñó e implementó GEOEDUCA, fue interdisciplinario y estuvo integrado por profesionales geógrafos, desarrolladores informáticos, diseñadores gráficos y educadores con especialización en Geografía. Durante un año, el grupo de profesionales geógrafos, en estrecha coordinación con los educadores especializados en geografía, analizaron las mallas curriculares para definir los contenidos que debían ser expresados mediante juegos y ejercicios.

Los diseñadores gráficos tuvieron la responsabilidad de esbozar interfaces que incorporaran dinamismo e interactividad con el usuario, para que finalmente los desarrolladores informáticos implementaran, a través de la web y en conformidad con los estándares, los interfaces creados por los grafistas.

El desarrollo de las interfaces, se basó en criterios que se definieron como básicos: simplicidad, animación, representación territorial, construcción de identidad, igualdad de género y plurinacionalidad. Estos dos últimos aspectos son considerados en Ecuador como prioritarios en sus políticas educativas, de manera que se puede decir que el proyecto está alineado con el principio constitucional del "Buen Vivir”, una visión del mundo, centrada en el ser humano como parte de un entorno natural y social, basado en los principios ancestrales del Sumak Kawsay (Díaz, 2016). 
La puesta en marcha de GEOEDUCA se realizó como parte complementaria de la IDE del IGM, convirtiéndose en pionera en el país. Su dirección electrónica es: $<$ http://www.geoportaligm.gob.ec/portal/index.php/geoeduca-inicio/>.

\section{GEOEDUCA, su estructura}

GEOEDUCA, considera tres grandes grupos de usuarios según su nivel de escolaridad:

- Educación básica, espacio dedicado a estudiantes de primero a décimo año de educación (5 a 14 años);

- Bachillerato, sitio con información para estudiantes de primero a tercer año de bachillerato (15 a 18 años) y

- Padres y docentes, lugar especializado con información para la guía y consulta.

Aunque cada espacio creado, tiene un diseño particular y personalizado que cubre con los requerimientos de cada grupo, todos tienen una misma lógica:

- Un área para aprender, que tiene información descriptiva y gráfica acerca de un tema específico;

- Una zona lúdica e interactiva para reforzar lo aprendido a través de juegos virtuales y experimentos; éstos van cambiando el nivel de dificultad conforme se cumplen los objetivos. Contiene herramientas intuitivas para ser usadas en el aula o para reforzar los conocimientos en el hogar; $y$,

- Un espacio para aplicar los conocimientos adquiridos y donde el alumno demostrará lo aprendido. Este espacio puede ser utilizado por el maestro como herramienta de evaluación dentro del aula o como tarea.

En el módulo de "Padres y Docentes" se encuentra información en línea de Biblioteca y Mapoteca, donde se puede descargar y consultar datos de diversos documentos científicos como atlas, artículos o guías, entre otros. En la Figura 1 se muestra su esquema estructural.

\section{Difusión y uso}

Siguiendo las recomendaciones de la Organización de las Naciones Unidas para la Educación, la Ciencia y la Cultura (UNESCO), sobre el acceso al conocimiento en el ciberespacio, se establecieron estrategias de difusión para el portal GEOEDUCA. Desde el año de su lanzamiento hasta el presente, se realizaron talleres participativos e incluyentes sobre su uso y aplicación en el aula, así como la importancia de utilizar fuentes oficiales de información. 


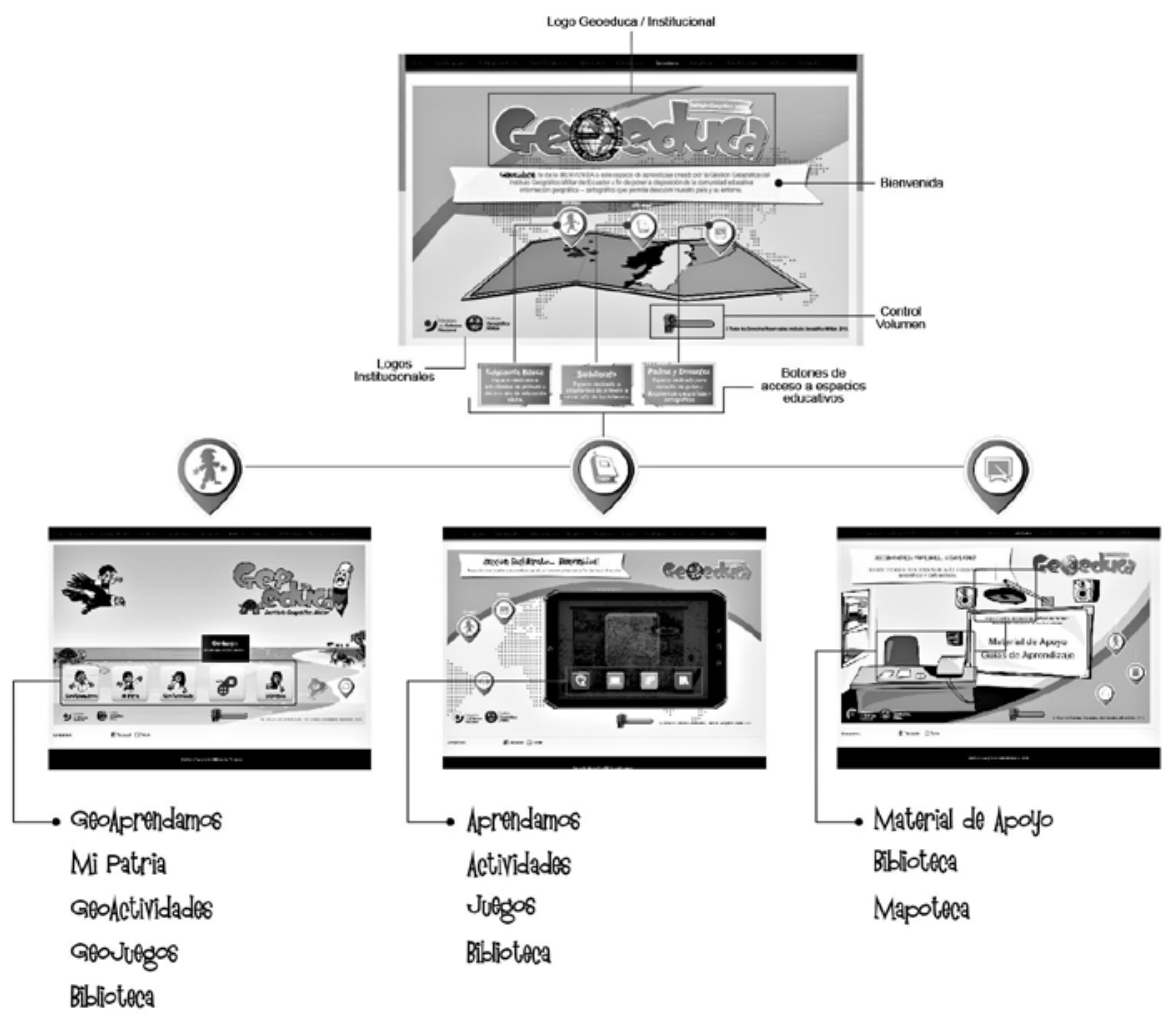

Figura 1. Esquema de GEOEDUCA. Autor: Xavier Vivas.

Estos espacios de interacción se ejecutan en dos partes: la primera, explica de forma conceptual su objetivo, brinda detalles y consejos prácticos para su mejor aprovechamiento. La segunda, es el acceso al geoportal, donde es posible interactuar, jugar y aprender. Para finalizar, los participantes tienen la oportunidad de colaborar con la iniciativa a través de sus comentarios y sugerencias.

Los talleres se han impartido anualmente, con el apoyo del Ministerio de Educación, de manera regular en todo el país, con la participación de más de 2,300 estudiantes de cuartos a séptimos años de educación básica (8 a 12 años) y 1,400 educadores de las asignaturas que componen el área de Entorno Natural, Social y Ciencias Sociales, véase Figura 2. Los centros educativos participantes han sido unidades públicas, municipales y privadas, tanto de las ciudades como de las zonas periféricas. De manera complementaria, se aprovecha que el IGM cuenta con un Centro Cultural, de acceso gratuito, conformado por un Planetario, salas astronómicas, aulas y museo interactivo que se integran con el proyecto GEOEDUCA y que los alumnos y educadores pueden visitar. 
A partir del año 2016 se integró al proceso de difusión el área de Vinculación con la Comunidad de la Universidad de Fuerzas Armadas (ESPE). En esta difusión intervienen estudiantes universitarios de las carreras de Ingeniería Geográfica y de Licenciatura en Educación; con este hecho se logró incrementar considerablemente el número de interesados en el proceso de divulgación, enseñanza y aprendizaje.

Hay que significar que el Ministerio de Educación del país, reconoció la relevancia de la iniciativa GEOEDUCA, con la integración de la misma a su portal de servicios educativos virtuales denominado "Educar Ecuador", que es un recurso en línea utilizado en las aulas o como fuente de consulta y apoyo.

\section{Número de personas capacitadas por año}

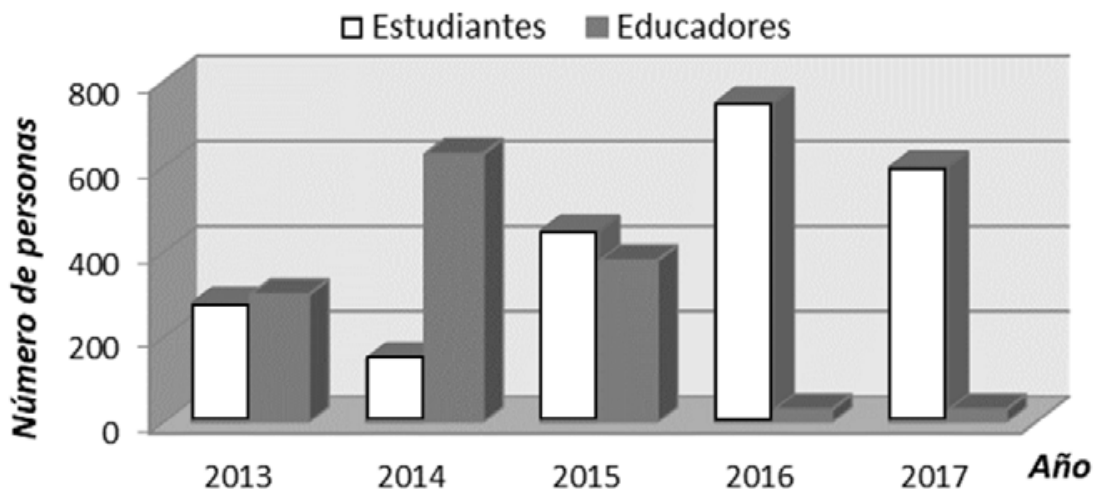

Figura 2. Número de personas capacitadas por año. Información recopilada de estadísticas del IGM, Ecuador. Realizado por las autoras.

\section{Resultados y expectativas}

El aporte más relevante de la iniciativa ecuatoriana GEOEDUCA, es el hecho de que la institución gubernamental responsable de la cartografía del país, haya puesto disponible su información oficial para el proceso de enseñanza-aprendizaje, en consideración de las necesidades particulares de un grupo de usuarios.

En el desarrollo de GEOEDUCA, se considera la importancia de facilitar a los estudiantes temas cartográficos-geográficos propios de su nivel educativo, provenientes de fuentes oficiales y que puedan ser utilizados como herramientas de trabajo en y para el aula.

La iniciativa GEOEDUCA, es un proyecto financiado por el IGM con grandes proyecciones para la educación ecuatoriana. Se tienen temas pendientes por desarrollar, principalmente en la sección para bachillerato, que demanda el diseño, la programación y la validación de los temas contenidos en la malla curricular. 


\section{Bibliografía}

Díaz Nafría, J.M. (2016). "Nuevos horizontes académicos para los estudios de tecnologías de la información y las comunicaciones en Ecuador”, repositorio digital Senescyt, <http://repositorio.educacionsuperior.gob.ec/handle/28000/3382>.

González, M.E.; Bernabé-Poveda, B.; Ángel, M.; Arcens, F.; Sánchez Hernández, J.; Capdevila Subirana, J. y Soteres Domínguez, C. (2010). La Infraestructura de Datos Espaciales como recurso educativo para el profesorado de la Educación Secundaria Obligatoria. Una propuesta innovativa de formación e-learning, Madrid, España.

León-Pazmiño, M.F.; Narváez-Benalcázar, R.; Bernabé-Poveda, M.A. y González Campos, M.E. (2016). “Geoinformación Institucional en el Ecuador: acceso y uso”, Revista Geoespacial, ESPE, vol. 13, núm. 1, Quito, Ecuador, pp. 40-58.

LOTAIP, Ley Orgánica de Transparencia y acceso a la información pública (2004). Ley 24, Registro Oficial Suplemento 337 de 18 de mayo del 2004, Quito, Ecuador. 